\title{
Existing and Future Standards for Event-Driven Business Process Management
}

\author{
Rainer von Ammon ${ }^{1}$, Christoph Emmersberger ${ }^{2}$, Thomas Ertlmaier ${ }^{1}$, Opher Etzion ${ }^{3}$, Thomas Paulus ${ }^{1}$, Florian Springer $^{2}$ \\ ${ }^{1}$ CITT GmbH, Konrad-Adenauerallee 30, D-93051 Regensburg, Germany \\ ${ }^{2}$ Senacor Technologies AG, Vordere Cramergasse 11, D-90478 Nürnberg, Germany \\ ${ }^{3}$ IBM HRL, Haifa University Campus, IL-31905 Haifa \\ \{Rainer.Ammon, Thomas.Ertlmaier, Thomas.Paulus\}@ citt-online.com, \{Christoph.Emmersberger, Florian.Springer\}@ senacor.com, \\ opher.etzion@il.ibm.com
}

\begin{abstract}
The development and operation of modern IT infrastructures requires generally accepted standards. Many standardization efforts are currently ongoing within the service oriented and event processing community. Recently a new discipline entitled "EventDriven Business Process Management (ED-BPM)" has emerged which takes a synergetic approach within this larger area. Consecutively, topics being discussed in this paper relate to the role of standards in the ED-BPM context, the need for a standard per se and the benefits and shortcomings of standardization in early phases vs. late phases. Within this activities, the most interesting proposal for a reference architecture is the Networked European Software and Services Initiative (NESSI) approach called NESSI Open Serivce Framework - Reference Architecture (NEXOF-RA) which has to be enhanced in order to support EDBPM applications. Thereupon a proposal for describing the context and structure of occurring events on a descriptive and non-complex level of events is discussed. Related industry standards like "Notification Event Architecture for Retail (NEAR)" and the possibility to extend that approach to other domains are surveyed. Two possible extensions are exemplified, for the logistics domain (NEAL), and for the finance domain (NEAF). Use cases for the retail, logistics, and finance domains are demonstrated. In the final analysis conclusions are drawn and proposed action items for advancing the ED-BPM standardization are made.
\end{abstract}

The following paper is a shortened version. The whole paper can be downloaded on the CITT-homepage http://www.cittonline.com/downloads/Existing_and_Future_Standards_for_EDB PM.pdf

\section{Submission Type \& Conference}

Industry Paper for the DEBS 2009 (short version), 3rd ACM International Conference on Distributed Event-Based Systems, July 6-9, 2009 - Nashville, TN, USA

Permission to make digital or hard copies of all or part of this work for personal or classroom use is granted without fee provided that copies are not made or distributed for profit or commercial advantage and that copies bear this notice and the full citation on the first page. To copy otherwise, or republish, to post on servers or to redistribute to lists, requires prior specific permission and/or a fee.

DEBS '09, July 6-9, 2009, Nashville, TN, USA.

Copyright 2009 ACM 978-1-60558-665-6/09/07... \$10.00.
General Terms

Standards

\section{Keywords}

ED-BPM - Event-Driven Business Process Management, Business Process Management, Complex Event Processing, Standards, Use Case, NEAL - Notification Event Architecture for Logistics, ISO - International Organization for Standardization

\section{INTRODUCTION TO}

\section{STANDARDIZATION EFFORTS}

The development and operation of modern IT infrastructures requires generally accepted standards. In large heterogeneous system landscapes, standards are indispensable for communication and data exchange. The benefit of standards can be characterized considering the following different types of involved adopters:

* meet the customers demand for interoperability

* standardized frameworks increase implementation speed

* reducing costs and risks for end users

However, it is not always the best solution for a young technology to develop a standard in early phases of its maturity, but without generating standards for new technologies, the innovation process can hardly be controlled which results in a rank growth of the technology [6].

The most interesting proposal for reference architecture is the Networked European Software \& Services Initiative (NESSI) [10] approach called NESSI Open Serivce Framework - Reference Architecture (NEXOF-RA) [11] shown in Figure 1 [12] which has been enhanced with event processing capabilities that are marked in each level of the layered architecture. Event processing may be layered itself to display increasing abstractions of events from a low level (technical layer) to higher level (business process layer) as proposed by David Luckham's event hierarchies [7]. 


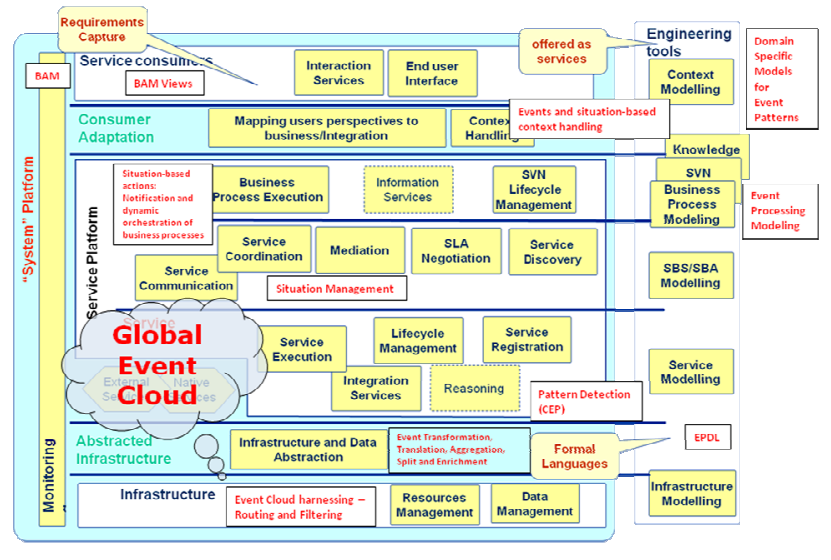

Figure 1. NEXOF-RA with event processing enhancements

A detailed explanation of the of the extensions of the existing NEXOF-RA is described in the long version of this paper.

The following extensions on the existing NEXOF-RA have been proposed in aspects of integrating event processing therein:

- Creating the Event Cloud

- Harnessing the Event Cloud

- Event Transformation

- Situation Management

- Actions - Events impact on the control flow

- Event processing modeling tools

- Semantic Language (EPDL - Event Processing Description Language)

- Event-driven Context handling

- The observation perspective (Business Activity Monitoring - BAM)

The amount, structure and interaction of events that are emitted inside this framework are much diversified. This leads to the common denominator in the form of similarities and diversities within and beyond business domains like automotive, aviation, logistic, entertainment, finance, banking, insurance or retail. The events, the patterns that can be derived, and the business processes in which they occur should be considered as a coherent part of the reference model, specific to every domain whereby issuing domain oriented Patterns (DoReMoPat) [2].

The modeling notation in the following use cases for the different domains does neither serve as a domain-specific language (DSL) being tailored to a specific application domain [8] nor inherits the capability of presenting the interaction, composition or aggregation of events. Rather, it is intended for describing the context and structure of occurring events on a descriptive and non-complex level hence it is composed by rudimentary elements derived from the Unified Modeling Language (UML) [15], the Models to Text Transformation Language (MOF) [14], and the XML Metadata Interchange (XMI) [13]. Additionally the notation is based on the IXRetail Notification for Event Architecture for Retail (NEAR) standard [9] which is discussed in detail in the next chapter.

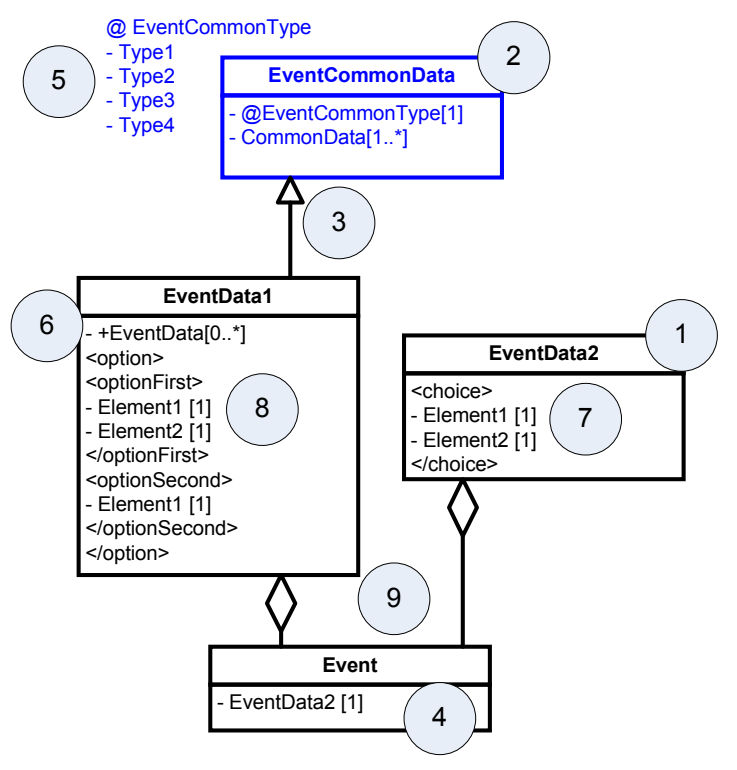

Figure 2. Exemplified modeling notation for the use cases

The modeling notation exemplified in Figure 2 contains the following elements:

1. The name of a data element is embedded in a rectangle whereas the color black is used by default.

2. Blue colored elements are determining common data elements that can occur multiple times within the domain model.

3. Lines with white arrowheads represent a generalization.

4. Content elements are indicated with a prefixed hyphen followed by their name and their possibility of occurrence in brackets in the data element.

5. The at-sign characterizes an attribute of the data element whose enumeration is decoupled from the rectangle and positioned beside it.

6. The plus sign indicates a composite element within a data element.

7. The keyword <choice> in greater-than and less-than signs determines the selection possibility of exactly one element from the group which can vary from instance to instance and ends with $<$ /choice $>$.

8. <option> indicates the grouping of elements in different configurations ending with a slash in front of the options name.

9. Lines with white diamond heads represent a generalization of choice in which exactly one choice per instance can occur.

\section{THE RETAIL DOMAIN AND THE NEAR STANDARD}

Figure 3 shows an example of a typical, distributed, heterogeneous IT infrastructure which can be found inside a retail enterprise. 


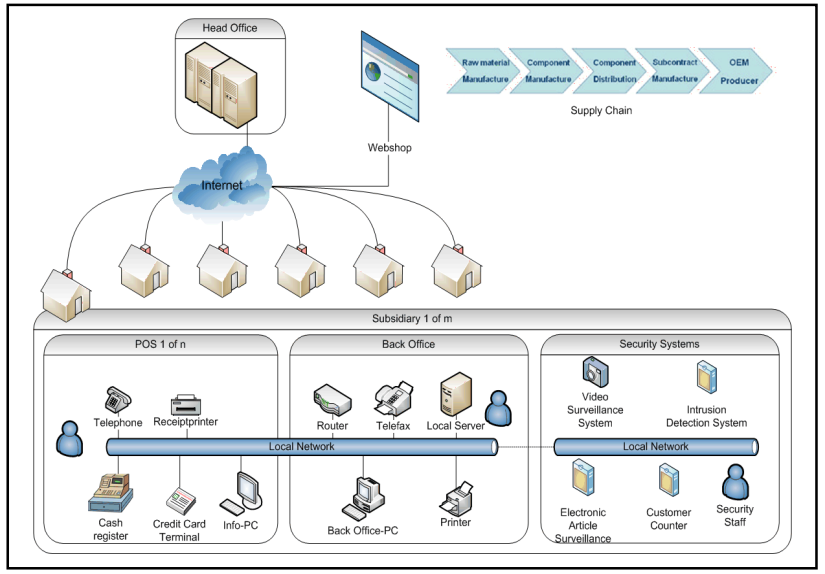

Figure 3. Typical organizational structure in retail industries

The head office is located on top of the typical organizational structure with the central IT backbone and the data center. All branches of the retail company are connected via the network to one large and complex enterprise bus. Inside the branches there is a wide variety of different devices and software systems from different vendors. To ensure proper communication between all different components, common communication standards are mandatory. A successful standard for processing events in the retail domain from the different devices and software systems is the IXRetail NEAR standard. This standard was designed and released by the Association for Retail Technology Standards (ARTS) of the National Retail Federation. The purpose of this standard is the reduction of time and cost for integrating retail applications by using asynchronous notification events. Therefore the standard defines a framework for XML formatted asynchronous notification event messages and the publish/subscribe methodology. It also defines a referencearchitecture to support the notification event framework and describes guidelines and best practices for future development and conformance testing.

From the business point of view the standard aims to simplify the real-time integration of loosely coupled applications and to lower the integration costs thus increase the customer service and operational efficiencies. The implementation of the reference architecture leads to an asynchronous, non persistent XML notification event architecture which removes the requirement for a direct point-to-point integration [3].

\section{EVENT MODEL OF THE NEAR STANDARD}

The Customer Event Domain Model describes a common data schema for dealing with customer activities. The attribute CustomerEvent holds the version of the model. This is a common element which can be found in all derived data models. The CustomerEventType describes the detailed event type. In this diagram two types of customer events are listed: CustomerIdentifiedEvent and CustomerAddedEvent. The EventCommonData are holding different technical information for processing this event in an appropriate context. The most interesting section in this model is the CustomerCommonData. This field shows the different attributes which have been defined by the IXRetail working group. It consists of rudimentary data which are necessary for processing customer data in the described retail domain.

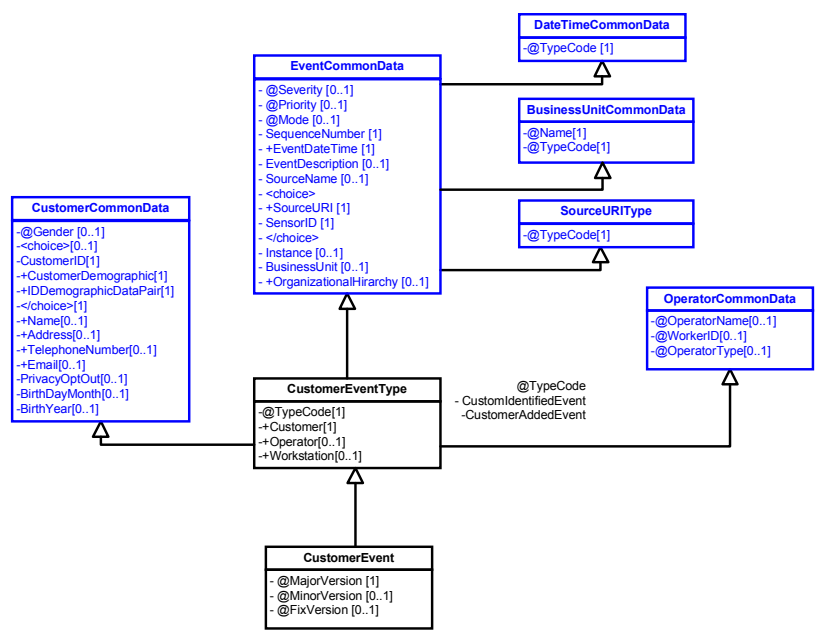

Figure 4. Customer Event Domain Model [3]

Future applications will not only act inside the enterprise, they will also interact beyond enterprise boundaries. Therefore the business information of the applications should be reusable in different domains. A simple case for such an application can be the event driven intercommunication between online retail stores and logistic companies. The customer information will be recorded and processed in the online store and have to be reused by the logistics company. For monitoring the payment status of an order process, the exchange of standardized data between the finance and the retail domain will also be necessary.

The shown data model from the NEAR standard is an adequate approach for processing customer events inside a single enterprise. But for cross-domain applications this model has to be extended to become a more common solution.

\section{NEAL: A LOGISTICS EVENTMODEL}

The general purpose of the Notification Event Architecture for Logistics (NEAL) is to support enterprises within the logistics domain in creating event driven systems for aligning their business and IT. The idea is to provide a basic set of events to assist in designing and implementing ED-BPM applications.

\subsection{Proposal: Logistics Event Architecture}

The creation of reference models requires both knowledge of the business processes and understanding of the events and their role. After this knowledge is established, complex events can be derived from the basis events to influence the processes in an automated, possibly preemptive way.

The main discipline in logistics is transportation. Its basic task is to move goods from a source to a sink. In the world of package deliverers, sources are generally the customers, sinks the recipients. Both, customer and recipient can be localized by their postal address. The postal address identifies both ends world wide unique, to enable the transportation from any customer to any recipient. Figure 5 shows an extract of the ISO/IEC 19773 postal address data structure in the notation of notification event architectures. For modeling event architectures of different domains, the idea is to take different common events. The Common Postal Address event is one of the candidates to become 
a common event over all domains. The reason for this is that address data is used in every domain and a common standard would increase the chance of an unproblematic data exchange.

Besides the "Postal Address Common Data" event several other patterns for the logistics domain are already developed, e.g. The "Order Event" or the "Tracking Event". The long paper gives a detailed description about these events.

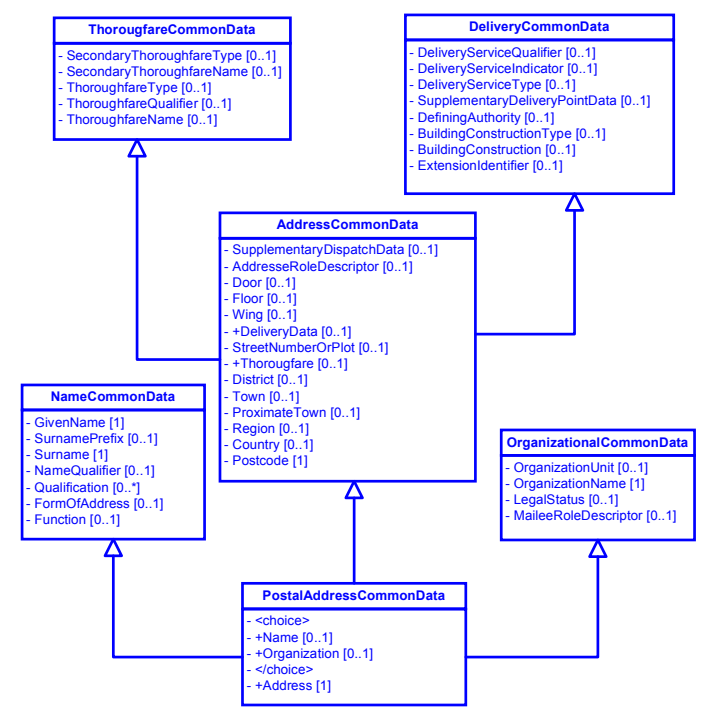

Figure 5. "Common Postal Address" Architecture derived from [12]

\section{NEAF - A POTENTIAL FINANCIAL EVENTMODEL}

The purpose of the Notification Event Architecture for Finance (NEAF) is to support enterprises within the finance domain in introducing event driven systems. The idea is to provide basic concepts in designing, implementing and managing event based systems.

The use of Complex Event Processing (CEP) within business processes promises, especially in the financial industry a great potential [5]. A bank is expected to introduce an adequate risk management to avoid situations emerging in the financial crisis. This requires on the one hand regulations and strategies providing policies for banks, and on the other hand technologies helping to get insight into complex processes. ED-BPM has the potential and technologies to provide a better insight into business processes and to allow reactions to potential exceptions before they actually occur [5]. Another use case with this approach is the detection of fraud [16]. A hurdle for the introduction of ED-BPM in finance and also other domains is that currently no standards, guidelines, or practical experience exists. This is necessary to allow a smooth and quick implementation of ED-BPM. The Notification Event Architecture for Finance (NEAF) aims to provide a set of reusable, standardized basic events and patterns tailored for finance business processes.

\subsection{Transactions as an Example for NEAF}

The first example of NEAF describes an account transaction or posting. For each money transfer, direct debit, cash withdrawal, car payments and many more, a transaction on the account of a customer has to be processed. The aim was to model a common and standardized event for an account transaction, which could be used in various business processes and for various banking institutes.

Fehler! Verweisquelle konnte nicht gefunden werden. describes the „AccountTransactionEvent“, which represents a common account transaction, as a first example of NEAF. The model is based on the notation introduced in chapter 1 . It consists on common data which are also necessary for other event models of NEAF. The "AccountTransactionType" is a template data model proposal for any kind of bank transactions. It is extended with common data of financial domain and specific data of business processes. It is a first attempt in describing basic events for EDBPM in the financial domain. In the future NEAF will provide a collection of templates and models helping to improve the introduction and management of event based systems for finance to improve design and implementation.

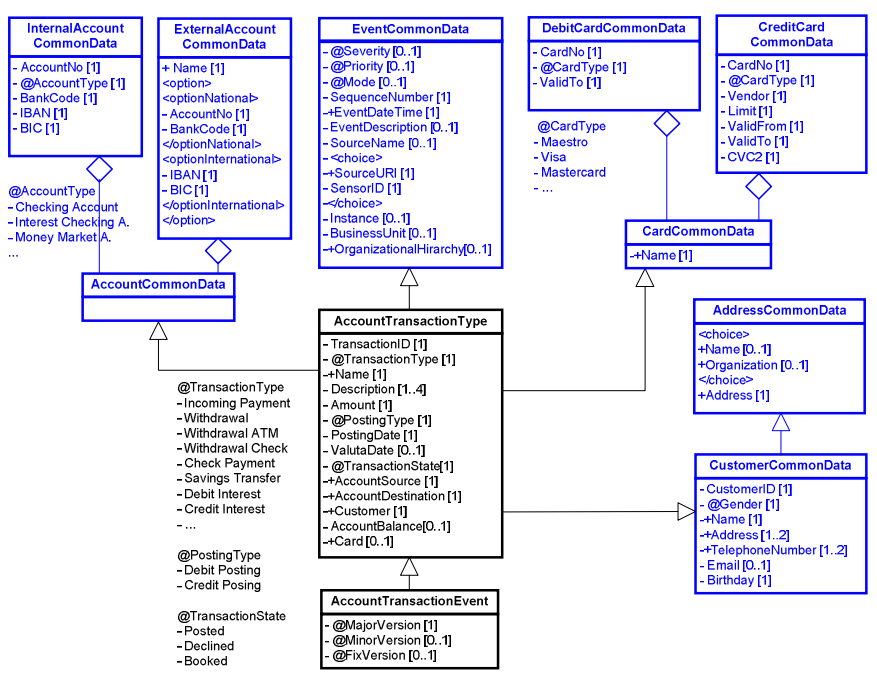

Figure 6: Model of the "AccountTransactionEvent" derived from [12]

\section{CONCLUSION AND FURTHER WORK}

The impact of the mentioned project ED-BPM/DoReMoPat is to show that the future internet of services on the basis of the EDBPM-reference model [1] and the enhanced NEXOF-RA allows to build applications much faster than today and allows to change respectively enhance and manage applications in a very flexible way. Detailed explanation of the project goals are explained in the long version of the paper. 


\section{REFERENCES}

[1] Ammon, R. v., Emmersberger, C., Springer, F., Wolff, C., 2008, Event-Driven Business Process Management and its Practical Application Taking the Example of DHL, 1st International Workshop on Complex Event Processing for Future Internet - Realizing Reactive Future Internet ( Vienna, Austria, September 28-30, 2008), FIS 2008, Retrieved February 26, 2009 from http://www.cittonline.de/downloads/FIS08_AmmonSpringer.pps

[2] Ammon, R. v., Silberbauer C., Wolff C., 2007, Domain Specific Reference Models for Event Patterns - for Faster Developing of Business Activity Monitoring Applications. VIP Symposia on Internet related research with elements of M+I+T++ (Lake Bled, Slovenia, October 8-11, 2007). Retrieved February 16, 2009 from http://www.cittonline.com/downloads/ReferenceModelsEventPatterns_with _Appendix_v3.pdf

[3] ARTS NRF, 2005, IXRetail Notification Event Architecture for Retail Charter, Retrieved February 13, 2009 from http://www.nrf.com/Attachments.asp?id=14056\&rid=509345

[4] ARTS NRF, 2006, Notification Event Architecture for Retail Schema, Retrieved February 13, 2009 from http://www.nrf.com/Attachments.asp?id=14022\&rid=795338

[5] DeLoach, D., 2008, CEP and the Banking Sector Outlook, Retrieved February 24, 2009 from http://blog.aleri.com/tag/banking/

[6] Gagliardi, F. 2009, Standardization Challenges, 1st European Conference on Software Services and SOKU technologies (Brussels, Belgium, January 13-14, 2009), SSOKU09 Retrieved February 14, 2009 from http://www.euecss.eu/contents/conference/ppt/ MSR_ECSS_Brussels_standards_Fab_v4.pdf/download

[7] Luckham, D., 2002, The power of events: An introduction to complex event processing in distributed enterprise systems, Addison Wesley Professional
[8] Mernik, M., Heering, J., Sloane, A., 2005, When and How to Develop Domain-Specific Languages, ACM Computing Surveys, Vol. 37, No. 4, December 2005. DOI= http://doi.acm.org/10.1145/1118890.1118892

[9] National Retail Federation, 2006, Press Release, Retrieved February 14, 2009 from http://www.nrf.com/modules.php ?name=News\&op=viewlive\&sp_id $=155$

[10] Networked European Software \& Services Initiative, 2005, NESSI Brochure, Retrieved February 15, 2009 from http://www.nessi-europe.eu/Nessi/Portals/0/NessiRepository/Publications/Flyers/2005_09_NESSI_Brochure.p df

[11] NEXOF RA, 2008, Definition of an architectural framework principles, Retrieved February 24, 2009 from http://www.nexof-ra.eu/sites/default/files/D7.2_Definition_ of_an_architectural_framework__principles.doc

[12] NEXOF RA, 2008, NEXOF Reference Architecture, Retrieved February 23, 2009, from http://www.nexofra.eu/?q=node/1

[13] Object Management Group, 2007, MOF 2.0/XMI Mapping, Version 2.1.1, Retrieved February 24, 2009 from http://www.omg.org/spec/XMI/2.1.1/PDF/index.htm

[14] Object Management Group, 2007, MOF Model to Text Transformation, Retrieved February 24, 2009 from http://www.omg.org/spec/MOFM2T/1.0/PDF

[15] Object Management Group, 2007, OMG Unified Modeling Language, Retrieved February 24, 2009 from http://www.omg.org/spec/UML/2.1.2/Infrastructure/PDF/

[16] Widder, A., Ammon, R. v., Schaeffer, P., Wolff, C., 2007, Identification of suspicious, unknown event patterns in an event cloud, ACM International Conference Proceeding Series; Vol. 233, Proceedings of the 2007 inaugural international conference on Distributed event-based systems DOI=http://doi.acm.org/10.1145/1266894.1266926 